\title{
FATORES DE RISCO PARA RETINOPATIA E HÁBITOS DE VIDA DO GRUPO HIPERDIA DA REGIÃO SUL
}

\section{RISK FACTORS FOR RETINOPATHY AND LIFESTYLE HABITS OF HIPERDIA GROUP SOUTHERN BRAZIL}

Geórgia Muccillo Dexheimer, Débora Konzen Scherer

\begin{abstract}
RESUMO
O objetivo deste estudo foi avaliar a associação entre fatores de risco, hábitos de vida e conhecimento que potencializam o desenvolvimento de Diabetes mellitus e Retinopatia Diabética. Participaram do estudo 31 usuários inseridos no grupo HIPERDIA, cuja finalidade é o acompanhamento de indivíduos com diabetes e hipertensão. Observou-se importante irregularidade na adesão dos usuários às práticas do grupo. Ainda, 29 participantes (93,55\%) apresentaram IMC aumentado, $28(90,3 \%)$ responderam que faziam uso de medicação para o controle da pressão arterial, 17 (54,8\%) apresentaram alteração da pressão arterial. Observou-se baixo nível de escolaridade, baixo conhecimento sobre sua patologia, controle e comprometimento com cuidados relacionados aos fatores de risco para retinopatia. Ressalta-se que através do melhoramento da atenção primária e do fortalecimento do autocuidado, pode-se melhorar a qualidade de vida dos pacientes diminuindo os riscos associados às patologias $\mathrm{e}$ os gastos públicos.
\end{abstract}

Descritores: Diabetes mellitus; Atenção Primária à Saúde; Fatores de Risco; Retinopatia diabética.

\section{ABSTRACT}

The study aimed to evaluate the association between risk factors, lifestyle habits and knowledge that potentiates the development of Diabetes mellitus and Diabetic Retinopathy. There were included 31 users enrolled in HIPERDIA group, whose purpose is to monitor individuals with diabetes and hypertension. There was an important irregularity in users' adherence to the practices of the group. Also, 29 (93.55\%) had increased BMI, 28 (90.3\%) said they were using medication to control blood pressure, $17(54.8 \%)$ had changes in blood pressure. It was found a low level of education, knowledge about their pathology and commitment with the required care according to risk factors to retinopathy. The improvement of primary care and strengthening self-care, patients' quality of life can be improved by reducing the risks associated with their pathologies and, consequently, reduce public expenditures.

Key words: Diabetes mellitus; Primary Health Care; Risk factors; Diabetic retinopathy. 
O Diabetes mellitus (DM) é uma doença considerada um problema de saúde mundial, uma vez que vem tomando proporções epidêmicas.1 Segundo a Sociedade Brasileira de Diabetes, atualmente estima-se que 6 a $7 \%$ da população brasileira é portadora do DM e acredita-se que este número pode dobrar até $2025 .{ }^{2}$ O DM está relacionado com mais de $40 \%$ das doenças cardiovasculares, configurando-se como doença crônica não-transmissível. No Brasil, dados de 2015 demonstram que o país está em quarto lugar em número de casos de diabetes, com mais de 14 milhões, perdendo apenas para Estados Unidos, Índia e China. Em 2015, foram registrados 130.700 óbitos resultantes do diabetes. Ainda, 0 grande número de complicações do DM que acometem os portadores, resulta em um elevado custo para a economia do país onde o gasto médio de um diabético, durante o ano para tratar a doença seja em torno de $\mathrm{R} \$ 4.848,50.3$

A longo prazo, o DM pode resultar em lesões crônicas, insuficiências em diversos órgãos, como por exemplo os olhos. Neste âmbito, pode-se citar agravos importantes relacionados à retinopatia que podem levar à cegueira quando não tratada. ${ }^{4,5}$ Dentre as alterações oculares que podem evoluir através do DM, a retinopatia ocupa $70 \%$ dos casos constituindo a principal complicação da doença. Cerca de 1 a 3\% da população mundial é acometida pela Retinopatia Diabética (RD), sendo responsável por 70 a $80 \%$ dos casos de cegueira neste grupo. ${ }^{6}$

0 desenvolvimento da RD pode estar associado a alterações genéticas e a fatores de risco ambientais ${ }^{7}$ Esta complicação pode ser relacionada a diversas patologias, porém, a maior incidência está presente no Diabetes tipo I, especialmente em pacientes que já convivem com a doença há anos, combinado ao controle ineficaz do nível glicêmico. A prevalência da RD é de $8 \%$ em pacientes com até 3 anos de diagnóstico, $25 \%$ para 5 anos, $60 \%$ para 10 anos e $80 \%$ para 15 anos do DM. -10 $^{-10}$ Além disso, outros fatores de risco associados à RD são: hipertensão arterial sistêmica (HAS), a qual apresenta maior frequência na população com DM; idade no momento do diagnóstico da diabetes; nefropatia diabética; sexo; tabagismo; dislipidemia e obesidade. ${ }^{11-19}$ Portanto, torna-se importante 0 acompanhamento de pacientes que apresentem DM e hipertensão quanto aos seus hábitos de vida e controle da doença.

Atualmente, os métodos de prevenção e tratamento para RD são falhos, devido a fatores múltiplos, como falta de médicos oftalmologistas na rede pública e orientação adequada aos pacientes, os quais desconhecem os seus agravos e, consequentemente, não realizam o acompanhamento correto para a sua patologia. ${ }^{20-22}$ Para evitar o surgimento das complicações secundárias, é necessário o diagnóstico precoce e campanhas de prevenção. Desta forma, o paciente poderá receber informações importantes sobre a progressão da doença e estabelecer um maior comprometimento com o tratamento e demais cuidados. ${ }^{23-26}$

A RD contribui para altas taxas de mortalidade e morbidade, constituindo uma grande ameaça para a saúde do usuário com DM, sendo o diagnóstico precoce o melhor caminho para postergar ou diminuir a sua progressão. ${ }^{27}$ Já fo demonstrado que a maioria da população desconhece a gravidade ou considera sua patologia sem gravidade. Isto reforça a ideia de que sem conhecimento não há adesão ao tratamento, e é de extrema importância que o sistema de saúde informe com mais clareza as técnicas comumente utilizadas. Além disso, um melhor planejamento da saúde pública do município permite um maior controle das patologias, onde o diagnóstico precoce destes agravos pode abrandar os custos da gestão pública com tratamentos e demais serviços. Além disto, este planejamento poderá refletir na melhora da qualidade de vida desta parte da população, que vem crescendo. ${ }^{21}$

Como estratégia para o controle do DM e hipertensão, desde 2002 foi implementado pelo Ministério da Saúde o programa Hiperdia (Sistema de Gestão Clínica de Hipertensão arterial e Diabetes mellitus da Atenção Básica). 0 programa tem por objetivo a qualificação do suporte de pacientes com estas patologias e 0 aumento da adesão ao tratamento, através do planejamento de ações de promoção à saúde. Portanto, são formados grupos de usuários que poderão participar de atividades que permitam o acesso à informação e autocuidado, controle de exames laboratoriais de rotina, práticas de atividades físicas e reeducação alimentar, sempre guiados por profissionais da área. ${ }^{22}$

Diante do exposto, este estudo teve como objetivo avaliar a presença de fatores de risco e sua associação com hábitos de vida relacionados ao desenvolvimento de retinopatia em uma população diabética usuária do grupo Hiperdia. Também foi avaliado o nível de conhecimento dos pacientes em relação à sua doença e seu comprometimento com as atividades requeridas para a manutenção da sua saúde.
Pesquisa de abordagem exploratória quantitativa e qualitativa realizada no grupo SisHiperdia (Sistema de Gestão Clínica de Hipertensão arterial e Diabetes mellitus da Atenção Básica) da Unidade Básica de Saúde de um município do interior do Rio Grande do Sul. O grupo realiza encontros quinzenais em quartas-feiras à tarde para receber orientação nutricional, física e acompanhamento médico, conforme programação preconizada pelo Ministério da Saúde. No período da realização do estudo estavam cadastrados neste grupo cerca de 60 participantes portadores de Diabetes mellitus.

\section{Coleta de dados}

Foram incluídos no estudo todos os participantes do grupo Hiperdia da Unidade Básica de Saúde avaliada Foram excluídos os pacientes que não aceitaram participar do estudo e que não compareceram aos encontros do grupo no período da coleta de dados nos meses de maio e junho de 2016

Foi aplicado um questionário com perguntas múltiplas relacionadas com os hábitos de vida dos participantes do grupo e informações pertinentes ao desenvolvimento da sua patologia. Posteriormente, foram analisados os valores do último exame laboratorial realizado em um período menor que 1 ano, avaliando resultados de glicose, colesterol $e$ triglicerídeos. A avaliação do Índice de Massa Corporal (IMC) foi realizada através da relação da massa corporal $(\mathrm{Kg})$ triglicerídeos. A avaliação do Indice de Massa Corporal (IMC) foi realizada atravess da relação da massa corporal $(\mathrm{Kg})$
e estatura $(\mathrm{m})$ ao quadrado utilizando-se critérios de classificação da Organização Mundial da Saúde onde valores de IMC iguais ou superiores a 25 são considerados acima do peso.29 A aferição da pressão arterial foi realizada após pelo menos cinco minutos de repouso do paciente utilizando-se um esfigmomanômetro (Aneroide G-TECH/Premium). Os valores da pressão arterial foram classificados de acordo com as Diretrizes Brasileiras de Hipertensão Arterial.30

O presente estudo foi aprovado pelo Comitê de Ética em Pesquisa da Instituição de Ensino UNIVATES, sob parecer de número 1.522.191 aprovado no dia 29 de abril de 2016. Todos os participantes do estudo leram e assinaram o Termo de Consentimento Livre e Esclarecido (TCLE) previamente à coleta de dados.

Metodologia de análise de dados

Os dados foram tabulados e analisados empregando-se o software SPSS (Statistical Package for the Social Sciences, IBM) versão 20.0. Foi realizado o teste de normalidade Shapiro-Wilk. Para amostras com distribuição normal, os resultados foram apresentados em média \pm desvio padrão e para amostras com distribuição não normal, os resultados foram apresentados em mediana (percentil 25 - 75). 0 nível de significância adotado foi de $5 \%$, sendo considerados significativos valores de $p \leq 0,05$.

RESULTADOS:

O grupo de usuários da Unidade Básica de Saúde analisada é constituído por cerca de 60 participantes. Foi observada uma baixa frequência de participação nos encontros durante o período avaliado. Portanto, foi possível aplica o questionário em apenas 31 pacientes, que assinaram previamente o TCLE. Os demais participantes não apresentaram frequência nas atividades do grupo no período de coleta dos dados.

Dentre as características sócio-demográficas da população de estudo, foi observada uma média de idade de 63,23 anos e baixo nível de escolaridade (Tabela 1).

Tabela 1. Características sócio-demográficas da população de estudo

\section{Característica Valor}

Idade*

Mínima

Máxima

Sexo

Feminino

$63,23( \pm 11,2)$

41

81

$24(77,4 \%)$ 


\section{Masculino $\quad 7(22,6 \%)$}

Escolaridade

\begin{tabular}{lc}
\hline Nenhuma & $3(9,7 \%)$ \\
$1^{\circ}$ grau incompleto & $22(71,0 \%)$ \\
$1^{\circ}$ grau completo & $2(6,5 \%)$ \\
$2^{\circ}$ grau completo & $4(12,9 \%)$ \\
Etnia & \\
Branco & $24(77,4 \%)$ \\
Pardo & $5(16,1 \%)$ \\
Negro & $2(6,5 \%)$ \\
\hline
\end{tabular}

Valores apresentados pela frequência numérica percentual. *Média e desvio padrão.

Além do questionário, foram analisados os últimos resultados dos exames laboratoriais, sendo considerados apenas os exames realizados no último ano. Um total de 25 pacientes $(80,7 \%)$ estavam de acordo com o período de 1 ano estabelecido pela Unidade Básica, sendo que $6(19,3 \%)$ pacientes não realizam exames laboratoriais com esta frequência.

São ainda menos frequentes os exames oftálmicos, onde $17(54,8 \%)$ pacientes realizam em períodos iguais ou superiores a 2 anos. Ainda, $29(93,55 \%)$ pacientes avaliados apresentam IMC elevado, sendo classificados como préobesos ou obesos (Tabela 2).

Tabela 2. Características clínicas da população de estudo

Característica

Valor

Diabetes mellitus

Tipo 1

Tipo 2

$30(96,8 \%)$

Frequência de exames laboratoriais

6 em 6 meses

Ano em ano

A cada 2 anos

Mais de 2 anos

Frequência de exames oftálmicos

6 em 6 meses

Ano em ano

A cada 2 anos

Mais de 2 anos

Mais de 5 anos

Nunca

IMC*

Normal $(18,5-24,9)$

Pré-obeso $(25,0$ a 29,9)

Obesidade Grau I (30,0 a 34,9)

Obesidade Grau II $(35,0$ a 39,9)

Obesidade Grau III $(\geq 40,0)$

Feminino

Masculino
$11(35,5 \%)$

$4(45,2 \%)$

$4(12,9 \%)$

$2(6,5 \%)$

$8(25,8 \%)$

$4(12,9 \%)$

$8(25,8 \%)$

$1(3,2 \%)$

$2( \pm 5,63)$

$8(25,81 \%)$

$12(38,71 \%)$

$5(16,13 \%)$

$4(12,90 \%)$

$32,34( \pm 6,01)$

$34,74( \pm 3,84)$

$\begin{array}{lc}\text { Colesterol }^{* *} & 191,47( \pm 29,78) \\ \text { Triglicerídeos }^{* *} & 152,38( \pm 30,5) \\ \text { Glicose }^{\star * *} & 163(138-178) \\ \text { Valor de Pressão Arterial (PAS/PAD) } & \\ \text { Ótima }(<120 /<80) & 10(32,3 \%) \\ \text { Normal }(<130 /<85) & 4(12,9 \%) \\ \text { Limítrofe }(130-139 / 85-89) & 5(16,1 \%) \\ \text { Hipertensão leve }(140-159 / 90-99) & 12(38,7 \%) \\ \text { Tempo de Diabetes (anos) } & \\ 1 \text { a } 10 & 20(64,5 \%) \\ 11-15 & 4(12,9 \%) \\ 16-20 & 4(12,9 \%) \\ 31-35 & 3(9,7 \%) \\ \end{array}$

Valores apresentados pela frequência numérica percentual. *IMC = Índice de Massa Corporal. Média e desvio padrão. ${ }^{* *}$ Média e desvio padrão com $n=19$. ${ }^{* * *}$ Mediana (percentil 25 - percentil 75 ) com $n=25$. PAS = pressão arterial sistólica. PAD = pressão arterial diastólica.

Todos os participantes do grupo eram diabéticos e $28(90 \%, 3 \%)$ hipertensos, os quais já faziam uso de medicação anti-hipertensiva. Desta forma, a pressão arterial foi mensurada com o objetivo de avaliar o controle da mesma pelos medicamentos. Foi constatado que, apesar do uso de medicação 12 (38,7\%) pacientes apresentaram valores de pressão arterial classificados como hipertensão leve. Ainda, apenas um paciente apresentou diagnóstico para retinopatia diabética. A avaliação da presença de fatores de risco para retinopatia na população estudada demonstrou que há um grande número de não praticantes de atividade física regular. Além disso, quase metade dos indivíduos disseram não ter cuidados com a alimentação (Tabela 3).

Tabela 3. Fatores de risco para retinopatia, medicamentos e hábitos de vida

\begin{tabular}{lcc}
\hline Característica & Sim & Não \\
\hline Tabagismo & $5(16,1 \%)$ & $26(83,9 \%)$ \\
Atividade física & $4(12,9 \%)$ & $27(87,1 \%)$ \\
Homens & - & $7(100 \%)$ \\
Mulheres & $4(16,7 \%)$ & $20(83,3 \%)$ \\
Cuidados com alimentação & $17(54,8 \%)$ & $14(45,2 \%)$ \\
Homens & $2(28,6 \%)$ & $5(71,4 \%)$ \\
Mulheres & $15(62,5 \%)$ & $9(37,5 \%)$ \\
Tratamento & & \\
Antidiabéticos & $30(96,8 \%)$ & $1(3,2 \%)$ \\
Insulina & $14(45,2 \%)$ & $17(54,8 \%)$ \\
Anti-hipertensivo & $28(90,3 \%)$ & $3(9,7 \%)$ \\
Antinflamatórios & $18(58,1 \%)$ & $13(41,9 \%)$ \\
Dificuldade de visão & $27(87,1 \%)$ & $4(12,9 \%)$ \\
\hline
\end{tabular}

Valores apresentados pela frequência numérica percentual.

Após a aplicação do questionário sobre o nível de conhecimento, foi observado que os usuários não apresentavam domínio completo sobre os cuidados e possiveis complicações relacionadas à sua patologia. 0 cuidado mais frequente foi associado com a alimentação, citado por $29(93,5 \%)$ pacientes. Pode-se destacar que $19(61,3 \%)$ pacientes não 
apresentaram conhecimento sobre possíveis complicações associadas ao DM. Acerca da alimentação, os pacientes apenas citaram que "não podem comer doce". As complicações associadas ao DM citadas por 12 (38,7\%) pacientes foram: "demora na cicatrização de feridas", "pode dar cegueira", "hereditário", "fundo emocional", "problemas no pé", "afeta rins, coração e visão", "pode perder membros do corpo". Ainda, 2 (6,5\%) relataram não apresentar nenhum conhecimento sobre o DM. Assim, pode-se observar que apesar das variadas atividades e palestras realizadas no grupo abrangendo discussões de diferentes assuntos com profissionais da área, não foi observado um nível de informação adequado sobre o DM e a importância dos cuidados para o seu controle.

Foi realizado o teste de regressão logística binária, a fim de avaliar covariações que poderiam estar associadas à dificuldade de visão. As covariáveis foram: fumante, tempo do DM, tratamento para DM, valor de pressão arterial, dificuldade de visão após o diagnóstico do DM, IMC, prática de exercícios físicos e cuidados com a alimentação. Não fo observada nenhuma associação na amostra estudada.

\section{DISCUSSÃo}

Na população estudada, 28 participantes $(90,3 \%)$ responderam que faziam uso de medicação para o controle da pressão arterial. Ainda, 12 (38,7\%) apresentaram uma pressão arterial classificada como hipertensão leve e $5(16,1 \%)$ apresentaram valores limítrofes, de acordo com o Ministério da Saúde.29 O total de pacientes diagnosticados com DM acima de 10 anos de diagnóstico, foi $11(35,5 \%)$. A mediana da glicose foi de $163(138$ - 178) e a média do IMC foi de $32,88( \pm 5,63)$, sendo que apenas $2(6,45 \%)$ apresentaram IMC normal, $8(25,81 \%)$ foram classificados como pré-obesos, $12(38,71 \%)$ com obesidade grau I, $5(16,13 \%)$ com obesidade grau II, e 4 (12,90\%) com obesidade grau III. Esses dados são condizentes com a literatura, uma vez que o DM tipo 2 esta associado com o sedentarismo e 0 excesso de peso. ${ }^{30}$ A partir destes resultados, pode-se observar que a população do estudo apresenta os fatores de risco associados a retinopatia. 8 -10,31-34

Não foi possível, neste estudo, relacionar os fatores de risco com a dificuldade de visão devido ao baixo número amostral. Um estudo realizado em um hospital Universitário avaliou 549 pacientes e demonstrou que a incidência de retinopatia foi maior em pacientes com a idade avançada, maior duração da diabetes, aumento da pressão arterial e mau controle metabólico ${ }^{35}$

Quanto ao hábito tabagista, o presente estudo observou que apenas $5(16,1 \%)$ pacientes eram tabagistas. No estudo realizado por Moreira e colaboradores, foi observado um índice elevado de pacientes fumantes, com $53,8 \%$, sugerindo a elaboração de novas estratégias de saúde a fim de diminuir hábitos de vida que podem agravar o estado da doença. ${ }^{36}$

Os participantes do estudo apresentaram uma baixa adesão à prática de atividade física, sendo que apenas 4 $(12,9 \%)$ relataram a realização de forma periódica. Esta prática foi relatada apenas por mulheres, sendo que do total de 7 homens, nenhum pratica atividades físicas. Em estudo realizado em Blumenau, foi observado que $78 \%$ dos pacientes eram sedentários, possivelmente em virtude das complicações, mas mesmo antes do diagnóstico da doença relataram não ter o hábito de praticar atividades físicas. ${ }^{37}$

O programa Hiperdia foi desenvolvido pelo Ministério da Saúde em 2002, a fim de melhorar o suporte dos pacientes hipertensos e diabéticos, a partir do planejamento de ações de promoção de saúde. Com isso, o plano sugere a formação de grupos com o objetivo de facilitar a adesão ao tratamento de suas patologias, controle dos exames laboratoriais de rotina, peso e pressão arterial, discussão de temas variados com palestras de profissionais de diferentes áreas e também, prática de exercícios físicos orientados. Além disso, são realizadas orientações para a reeducação alimentar através de acompanhamento nutricional.22 Apesar destas atividades ocorrerem de forma regular na unidade Básica de Saúde avaliada, $29(93,5 \%)$ relataram cuidados com a alimentação e destes pacientes, apenas $4(13,8 \%)$ relataram questões específicas da alimentação, como o cuidado na ingestão de carboidratos. 0 restante relatou apenas cuidados em relação à ingestão de doces, como refrigerantes, tortas e chocolate. Estas informações são indicadores que devem ser avaliados para o controle da glicemia e obesidade, que são fatores de risco importantes para a retinopatia. Como relata Pereira e colaboradores, os hábitos de vida, alimentação inadequada e sedentarismo são os principais fatores para o desenvolvimento da obesidade. ${ }^{38}$

Diante do baixo nível de escolaridade e conhecimento geral da doença relatado pelos pacientes, deve-se avaliar os métodos utilizados pelos profissionais do grupo da Unidade Básica de Saúde, a fim de verificar o grau de entendimento destes pacientes em relação às informações repassadas a eles e cuidados a serem tomados mediante a sua doença. A prevenção primária é importante para que o processo de adoecimento seja controlado, permitindo investimento na promoção da saúde para diminuição das doenças crônicas e diminuição de custos por agravos secundários ${ }^{39}$ Segundo a Federação Internacional de Diabetes, o custo para o tratamento de pacientes com DM é de duas a três vezes maior do que pacientes não diabéticos, 3 o que torna importante a implantação de programas que visem o controle da doença $e$ contribuam para a diminuicão dos custos.

Em uma revisão bibliográfica de estudos epidemiológicos sobre DM, relatou-se que o desenvolvimento desta doença está relacionado com o sobrepeso e indivíduos sedentários. Além disso, foi observado um menor nível de escolaridade dos indivíduos.40 No presente estudo, também foi observado um nível baixo de escolaridade, sendo que apenas $4(12,9 \%)$ apresentaram segundo grau completo e $2(6,5 \%)$ com primeiro grau completo. 0 nível de escolaridade é uma característica importante para o entendimento da gravidade da doença. É importante direcionar 0 assunto programado destes grupos de uma forma acessivel, que todos possam entender.36 Em estudo realizado por Almeida et al. também foi observada a baixa escolaridade relacionada ao tempo da doença. A baixa escolaridade ou a falta de estudos delimita o entendimento da doença e seu tratamento correto. ${ }^{41}$ Pacientes diabéticos analisados apresentaram uma escolaridade baixa ou nula e, ainda, $61,5 \%$ dos pacientes entrevistados não apresentaram conhecimento sobre sua doença antes do diagnóstico. ${ }^{35}$

O grupo Hiperdia avaliado apresenta cerca de 60 participantes inscritos. Porém, estes participantes não frequentam os encontros com regularidade, sendo que no período de coleta dos dados, observou-se uma frequência de 20 participantes por encontro, havendo uma variação entre eles. Isto demonstra uma baixa participação do grupo e comprometimento. Isto também pode ser observado em relação a outras atividades desta população, onde 25 $(80,7 \%)$ pacientes realizam exames laboratoriais regularmente (ano em ano). Com relação aos exames oftálmicos, 0 comprometimento é inferior, sendo que apenas $10(32,3 \%)$ realizam de forma periódica (ano em ano). A prevenção, diagnóstico precoce e tratamento adequado, são essenciais para a qualidade de vida do paciente e, consequentemente, para a redução dos gastos públicos e diminuição do número de admissões hospitalares. ${ }^{33}$

Diante do baixo nível de escolaridade apresentado neste grupo, do baixo conhecimento da sua patologia e, consequentemente, baixo comprometimento com os cuidados que a doença exige, é possível observar que não há um controle regular da doença nestes indivíduos. Também não é possível observar um trabalho preventivo para possíveis complicações secundárias como a retinopatia. Portanto, é importante que sejam revistos os métodos empregados pelos profissionais de grupos de atenção primária, para que haja um melhor aproveitamento destes encontros realizados no grupo Hiperdia, a fim de fortalecer 0 autocuidado através do comprometimento dos pacientes. Isto torna o serviço de saúde pública mais eficiente, evitando problemas de saúde secundários que geram aumento dos gastos públicos e diminuem a qualidade de vida.

Considerações Finais

A retinopatia é uma doença grave e ainda muito frequente na população, exigindo um cuidado especial das equipes de saúde pública para evitar o seu desenvolvimento em grupos de risco, ou mesmo, a sua progressão. Apesar de não ser possível, neste estudo, relacionar a dificuldade de visão diretamente com os fatores de risco avaliados, foram observadas limitações no controle da DM e hipertensão bem como limitações na adesão aos cuidados com a sua condição. Além disso, o nível de conhecimento sobre a patologia foi limitado. Vale ressaltar que a falta de informação é crucial para a falta de comprometimento no tratamento de qualquer doença. Desta forma, torna-se essencial o acesso à informação para um bom controle do DM e comprometimento com os cuidados relacionados aos hábitos de vida bem como a realização de exames periódicos para o diagnóstico precoce. Isto pode contribuir para a prevenção do surgimento de complicações secundárias como a retinopatia, além de melhorar a qualidade de vida dos pacientes.

Os grupos de atenção primária devem ser compostos por uma equipe multiprofissional, e sugere-se a realização de uma avaliação periódica das atividades e assuntos discutidos nos encontros, a fim de verificar se os participantes apresentam um bom entendimento e comprometimento. Ainda, através do melhoramento da atenção primária e do fortalecimento do autocuidado, pode ser possível, além da melhora da qualidade de vida dos pacientes, a diminuição de 
gastos públicos devido ao controle da doença.

\section{Referências}

1. NCD Risk Factor Collaboration. Worldwide trends in diabetes since 1980. The Lancet. 2016 Apr 2.

2. Brasil. Diretrizes da Sociedade Brasileira de Diabetes: 2013-2014. Sociedade Brasileira de Diabetes, São Paulo: AC Farmacêutica, 2014. Disponível em:< http://www.nutritotal.com.br/diretrizes/files/342--diretrizessbd.pdf>.

3. International Diabetes Federation. IDF Diabetes Atlas. 7th ed., Brussels, Belgium: International Diabetes Federation, 2015. Disponível em: < www.idf.org/diabetesatlas>

4. Figueira CFC. Epidemiologia da retinopatia diabética na diabetes mellitus tipo 1 [dissertação]. Faculdade de Medicina da Universidade de Coimbra. 2010.

5. Aiello LP, DCCT/EDIC research group. Diabetic retinopathy and other ocular findings in the diabetes control and complications trial/epidemiology of diabetes interventions and complications study. Diabetes care. 2014 Jan 1;37(1):1723.

6. De Almeida BA, Yamasato JM, Medeiros CM. Direção veicular em indivíduos com retinopatia diabética: revisão e reflexão sobre as normas atuais. Saúde, Ética \& Justiça. 2015, 20(1): 21-28.

7. Esteves J, Laranjeira AF, Roggia MF, Dalpizol M, Scocco C, Kramer CK et al. Fatores de Risco para Retinopatia Diabética. Arq Bras Endocrinol Metab. 2008; 52(3):431-41.

8. Ting DS, Cheung GC, Wong TY. Diabetic retinopathy: global prevalence, major risk factors, screening practices and public health challenges: a review. Clinical \& experimental ophthalmology. 2016 Jan 1.

9. Yau JW, Rogers SL, Kawasaki R, Lamoureux EL, Kowalski JW, Bek T. Global prevalence and major risk factors of diabetic retinopathy. Diabetes Care. 2012 Mar; 35(3):556-64.

10. Ajvazi A, Lutaj P, Goranci I. Management of diabetic retinopathy, prevalence and clinical classification. Oftalmologia (Bucharest, Romania: 1990). 2013 Dec;58(4):47-50.

11. Klein R, Knudtson MD, Lee KE, Gangnon R, Klein BE. The Wisconsin Epidemiologic Study of Diabetic Retinopathy XXII: the twenty-five-year progression of retinopathy in persons with type 1 diabetes. Ophthalmology. 2008 Nov 30;115(11):1859-68.

12. Namperumalsamy P, Kim R, Vignesh TP, Nithya N, Royes J, Gijo T, Thulasiraj RD, Vijayakumar V. Prevalence and risk factors for diabetic retinopathy: a population-based assessment from Theni District, south India. British Journal of Ophthalmology. 2009 Apr 1;93(4):429-34.

13. Tanuja A, Guruprasad BS, Prashanth K, Prasad I. Prevalence and risk factors of diabetic retinopathy in a rural population of South India. International Journal of Diabetes in Developing Countries. 2015 Nov 1;35(3):356-61.

14. Abougalambou SS, Abougalambou AS. Risk factors associated with diabetic retinopathy among type 2 diabetes patients at teaching hospital in Malaysia. Diabetes \& Metabolic Syndrome: Clinical Research \& Reviews. 2015 Jun 30;9(2):98-103.

15. Cade WT. Diabetes-Related Microvascular and Macrovascular Diseases in the Physical Therapy Setting. Physical Therapy. 2008;88(11):1322-1335.

16. American Diabetes Association. Standards of Medical Care in Diabetes-2017 Abridged for Primary Care Providers. Clinical Diabetes. 2017 Jan 1;35(1):5-26.

17. Gholamhossein Y, Behrouz H, Asghar Z. Diabetic retinopathy risk factors: plasma erythropoietin as a risk factor for proliferative diabetic retinopathy. Korean Journal of Ophthalmology. 2014 Oct 1;28(5):373-8.

18. Sosna T, Svancarová R, Netuková M. Risk factors for diabetic retinopathy. Vnitrni lekarstvi. 2013 Mar;59(3):177-

81.

19. Tan G, Kamran Ikram M, Y Wong T. Traditional and novel risk factors of diabetic retinopathy and research challenges. Current medicinal chemistry. 2013 Sep 1;20(26):3189-99.

20. Castagno VD, Fassa AG, Silva MC, Carret MLV. Carência de atenção à saúde ocular no setor público: um estudo de base populacional. Cad. Saúde Pública, Rio de Janeiro, 2009 Oct; 25(10):2260-2272.

21. Silva VB, Temporini ER, Moreira Filho DD, Kara-José N. Tratamento da retinopatia diabética: percepções de pacientes em Rio Claro (SP)-Brasil. Arq Bras Oftalmol. 2005 Jun 1;68(3):363-8. 